\title{
Quantitative MRI in Cancer
}

\author{
T.E. Yankeelov, D.R. Pickens, and R.R. Price, eds.
}

Boca Raton, FL: CRC Press, 2011, 312 pages, $\$ 129.95$

Cancer is the result of genetic and other changes that lead to alterations in normal cellular function, resulting in unlimited growth and expansion that occur both locally and at distant sites within the body. Advances in the science and technology of medical imaging and radiation therapy are complex, computer-driven, and more profound and rapid than ever before. A wide variety of imaging platforms is currently used for both clinical assessment and preclinical studies. A current trend in the clinical practice of oncology is to use a combination of modalities to assess tumors, particularly staging and evaluation of therapeutic response.

Recent years have seen a tremendous explosion in both the number and the quantity of imaging techniques that can be applied in the quantitative characterization of cancer. Relevant techniques that have been developed report on tumor cellularity, vessel perfusion and permeability, hypoxic fractions, and cellular and molecular signatures. MRI is currently one of the more powerful techniques available in medical imaging, and quantitative MRI technique can be moved from bench to bedside.

This book is from the series entitled "Imaging in Medical Diagnosis and Therapy," edited by Dr. William R. Hendee. The goal of this book is to provide a 1-volume overview of the theoretic and experimental essentials of MRI. This book consists of 5 parts with 22 chapters. Part 1 deals with the physical basis of MRI and has 4 chapters related to biology and imaging of cancer, MRI physics, hardware and data acquisition of MRI, and image formation. Part 2 discusses the characterization of tissue properties with endogenous contrast mechanisms and has 5 chapters dealing with quantitative measurement of $\mathrm{T} 1, \mathrm{~T} 2, \mathrm{~T} 2 *$, and proton density; arterial spin labeling techniques; diffusion MRI; magnetization transfer and chemical exchange saturation transfer imaging in cancer; and MR spectroscopy and spectroscopic imaging of tumor physiology and metabolism. Part 3 addresses the characterization of tissue properties with ex- ogenous contrast agents and has 5 chapters handling contrast agents for T1-weighted MRI, nanoparticles for T2- and T2*-weighted MRI, dynamic contrast MRI, dynamic susceptibility MRI, and MR angiography. Part 4 covers imaging processing in cancer, with 5 chapters related to MRI of tissue oxygenation status, clinical assessment of the therapeutic response of tumors with MRI, image segmentation, spatial and temporal image registration, and synthesis of multiparametric data. Part 5 is dedicated to emerging trends and has 3 chapters dealing with MRI in radiation therapy planning, molecular and cellular imaging, and hyperpolarized MRI of cancer.

This book presents a range of quantitative MRI methods for assessing tumor biology, including biophysical and theoretic explanation of the most relevant MRI techniques as well as examples of these techniques in cancer applications. A more quantitative (and potentially more pathologically specific) approach to tissue modeling is necessary to separate the contrast due to magnetization transfer from that due to relaxation. A goal of quantitative studies of chemical exchange saturation transfer is to isolate the underlying biophysical changes that occur in pathology. There have been several studies that quantified metabolite concentration or exchange by determining signal ratios of multiple agents for chemical exchange saturation transfer. It is of note that there is no dedicated chapter on quantification of MRI. Images are clear and instructive, although the paper is not glossy. References are updated.

I recommend this special book to trainees and radiologists using advanced MRI and to basic scientists and physician scientists using MRI.

\author{
E. Edmund Kim \\ M.D. Anderson Cancer Center \\ 1400 Pressler, Unit 1483 \\ Houston, TX 77030 \\ E-mail:ekim@di.mdacc.tmc.edu
}

